\title{
ACCELERATION OF THE SOLAR WIND: A NEW VIEW
}

\author{
V.H. HANSTEEN AND E. LEER \\ Institute of Theoretical Astrophysics \\ PB 1029 Blindern, 0315 Oslo, Norway
}

\section{Introduction}

The last few years have seen the development of solar wind models characterized by high coronal proton temperatures, low electron temperatures and low densities in the inner corona. In addition the emphasis on "additional" acceleration via Alfvén waves has been muted and been replaced by models where the acceleration is essentially complete within $10 R_{\odot}$. This work has, in part, been spurred by Ulysses observations of the high speed wind and by the expectations of the observations to be made by the various instruments on the SOHO satellite. In this paper we show how these new models differ from earlier ones.

Our current understanding of the solar wind is based upon the work of E.N. Parker in the late 1950-s and early 1960-s. Parker (1958) described the expansion of an isothermal fully ionized hydrogen corona into a supersonic solar wind. Let us briefly review this solution as it is relevant to the discussion to be carried out below. The particle flux in an isothermal, spherically symmetric electron-proton solar wind can be found analytically. It is determined primarily by

$$
n_{\mathrm{c}}=n\left(r_{\mathrm{c}}\right)=n_{0} \exp \left(-\frac{G M_{\odot} m}{2 k T r_{0}}+\frac{3}{2}\right)
$$

the electron density at the critical point at $r_{\mathrm{c}}=G M_{\odot} m / 4 k T$ where the velocity is equal to the thermal velocity $u_{\mathrm{c}}=\sqrt{2 k T / m}$ and where $n=n_{0}$ at some "coronal base" $r=r_{0}$. Notice that the density at the critical point only differs from that of a static corona by a small factor; the density structure in the subsonic portion of a wind solution is essentially the same as that in a static model. Since we know the location, density and velocity at the critical point we can, given the coronal temperature and base density, predict the proton flux emanating from the Sun.

$$
\frac{(n u)_{\mathrm{E}}}{10^{12} \mathrm{~m}^{-2} \mathrm{~s}^{-1}}=0.4\left(\frac{n_{0}}{10^{14} \mathrm{~m}^{-3}}\right)\left(\frac{T}{10^{6} \mathrm{~K}}\right)^{3 / 2} \exp \left[-11.55\left(1-\frac{10^{6} \mathrm{~K}}{T}\right)\right] \text {. }
$$

As is readily seen, the proton flux is proportional to the coronal base density $n_{0}$ and increases exponentially with increasing temperature, $T$. The sensitive dependence of the proton flux with coronal temperature makes it possible to construct solar wind models with reasonable proton fluxes for virtually 'any' value of the coronal base density, $n_{0}$. Observations of the coronal temperature (Withbroe, 1988) give no reason to assign the corona a constant temperature and are therefore consistent with large variations of the solar wind proton flux. On the other hand in situ observations show that the solar wind proton flux is fairly constant (McComas et al., 1995). This appear-ant discrepancy between theory and observation constitutes 'the solar wind mass flux problem' (Leer and Holzer, 1991).

\section{Self consistent corona/solar wind models}

In the traditional models discussed above the density, $n_{0}$, and the temperature, $T_{0}$, at the coronal base are specified and considered as independent parameters, i.e. one starts with a heated inner solar corona and assumes that coronal energetics are not perturbed by the presence of a wind. It is this assumption that recent studies have shown to be flawed as we will illustrate below. In most 

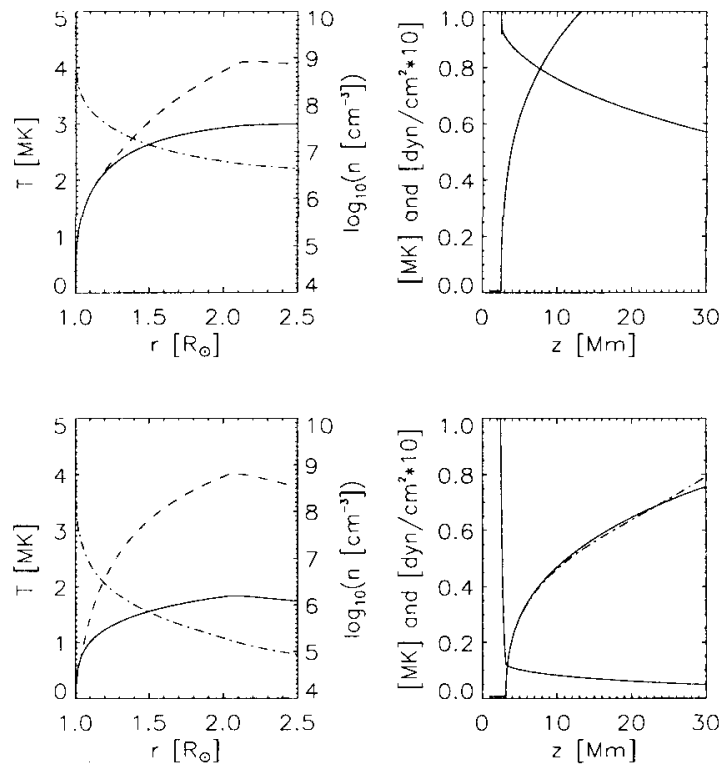

Figure 1. A comparison of spherically symmetric static and wind solutions for the same mechanical energy input. In the static case, shown in panels (a) and (b), the flow speed and the temperature gradients are set to zero at $r=2.5 R_{\odot}$. For both the static and wind solutions, which are shown in panels (c) and (d), the energy input is near $r=2 R_{\odot}$ (viz, $r_{1}=2.0 R_{\odot}, h_{M}=0.1 R_{\odot}$, and $f_{M 0}=1.5 \times 10^{5} \mathrm{erg} \mathrm{cm}^{-2} \mathrm{~s}^{-1}, 60 \%$ is inserted in the proton fluid and $40 \%$ in the electron fluid). The horizontal scale in panels (b) and (d) is in megameters, in order to show the upper chromosphere and transition region, while the horizontal scale in panels (a) and (c) is in solar radii, in order to show the corona. In the coronal panels, the proton density is shown by a dot-dash line and the electron and proton temperatures by solid and dashed lines, respectively. In the transition region panels, the pressure and electron temperature are shown by solid lines, while the proton temperature is shown by a dot-dash line.

cases the solar wind consumes a large portion of the energy dissipated in the corona, thereby also determining a relationship between the coronal base density and the coronal temperature.

Consider models where the formation of the corona and the acceleration of the solar wind are treated as a coupled problem. In this approach, pioneered by Hammer $(1982 \mathrm{a}, \mathrm{b})$ and by Withbroe (1988), the parameters that determine the model are set by the processes assumed to heat the corona. For the purposes of discussion we consider a coronal heating consistent with a "mechanical" energy flux that varies as

$$
\begin{aligned}
& F_{\mathrm{m}}=F_{\mathrm{m} 0} \text { for } r<r_{\mathrm{m}} \\
& F_{\mathrm{m}}=F_{\mathrm{m} 0} \exp \left[-\left(r-r_{\mathrm{m}}\right) / L_{\mathrm{m}}\right] \text { for } r \geq r_{\mathrm{m}}
\end{aligned}
$$

where $F_{m 0}$ is the amplitude at the base of our model, $L_{\mathrm{m}}$ is the damping scale length, and $r_{\mathrm{m}}$ the point where the damping sets in. With this type of coronal heating function we can vary the amplitude, position and scale height of heat insertion. For multi-fluid models we can also vary the apportionment of energy between various particles species. The coronal density and temperature are set by the interplay between the energy requirements of solar wind acceleration, conductive heat flux losses to the transition region/chromosphere and the radiative losses occuring there.

To illustrate the connection between coronal heating, solar wind acceleration, conductive losses through the transition region and the coronal density let us consider a numerical study, in which, for the same mechanical energy input, a static atmosphere (magnetically contained) and an expanding atmosphere (i.e., a wind) are compared. The results of this study are shown in Figure 1. The principal difference in these two cases arises from the fact that in the static atmosphere, all of the mechanical energy flux deposited in the corona is conducted back to the chromosphere and radiated 
away, whereas in the expanding atmosphere, virtually all (i.e., some $90 \%$ ) of the mechanical energy flux deposited in the corona is carried away by the wind. In the former case the chromospheric density at the base of the transition region must be large in order to radiate away the energy conducted down as can be seen in the right hand panels. Since the pressure scale height is long compared to the temperature scale height in the transition region this implies that also transition region and coronal densities are much (10 times) higher in this model. The transition region panels also show a much steeper temperature gradient which reflects the considerably larger heat flux that is transported inward to the chromosphere. In the coronal panels, on the left hand side of Figure 1 both the electron temperature and the density are seen to be higher in the static case, and the electron and proton temperatures are coupled to higher altitudes, owing to the higher density.

\section{Energy balance in the solar wind}

In an extensive parameter study Hansteen and Leer (1995) show that unless coronal heating occurs close to the sun, within a coronal scale height or so, almost all the input energy invariably goes into accelerating the solar wind. Though the variations in the location and particle species the mechanical flux is dissipated into produce quite distinct coronal structures it is remarkable that all the models have approximately the same solar wind mass flux. The key to understanding this small variation in mass flux lies in considering the energy balance of the consistent chromosphere - transition region - corona -solar wind models. For illustration let us consider the energy balance for an electron - proton fluid

$$
\rho u A\left(\frac{1}{2} u^{2}+\frac{\gamma}{\gamma-1} \frac{p}{\rho}-\frac{G M_{\odot}}{r}\right)+F_{\mathrm{q}}+F_{\mathrm{m}}+F_{\mathrm{Rad}}=F_{\epsilon},
$$

where $\rho=m n$ ( $m$ is the proton mass), $\gamma$ is the ratio of the specific heats $c_{p} / c_{v}$, and $p=2 n k T$ ( $k$ is Boltzmann's constant) in a one-fluid model. The conductive heat flux is $F_{q}=A q$ and the radiative flux $F_{\mathrm{Rad}}=\int_{0}^{r} A n_{\mathrm{e}} n_{\mathrm{H}} f\left(T_{\mathrm{e}}\right) d r$. Noting that the energy flux $F_{\varepsilon}$ is conserved in the flow we rewrite Eq. (4) comparing the energy fluxes at the base of our model, subscript ' 0 ' where the energy flux resides mainly in gravitational potential energy and in the input mechanical energy flux, and at a distance far from the Sun, subscript ' $\infty$ ' where the energy flux in this high Mach number flow is comprised primarily of the solar wind kinetic energy flux plus whatever of the input energy flux that may have gone into radiation

$$
F_{\mathrm{m} 0}+\mathcal{F}\left(-\frac{1}{2} v_{\mathrm{g}}^{2}\right) \approx \mathcal{F}\left(\frac{1}{2} u_{\infty}^{2}\right)+F_{\mathrm{Rad}, \infty}
$$

with $\frac{1}{2} v_{\mathrm{g}}^{2}=G M_{\odot} / R_{\odot}$. These relations show that in the expanding solar wind the input mechanical energy flux, mediated by the conductive heat flux, is used to lift the gas out of the solar gravitational field and accelerate the flow to its asymptotic flow speed, $u_{\infty}$, a certain percentage of the input energy flux flows back into the chromosphere where it is radiated away. Hansteen and Leer (1995) find that in the parameter regime represented by these models the majority $(\approx 80-90 \%)$ of the available energy flux goes into lifting coronal gas to infinity and to accelerating it to high speed, thus

$$
\mathcal{F} \approx \frac{F_{\mathrm{m} 0}}{\frac{1}{2} v_{\mathrm{g}}^{2}+\frac{1}{2} u_{\infty}^{2}}
$$

This explains the similarity in the mass loss rates for almost all models. Since most of the energy goes into driving the wind and since $u_{\infty} \approx v_{\mathrm{g}}$ for all the models the mass flux rate is proportional to the input energy flux in the corona.

\section{Obtaining a high speed wind}

Having produced a plausible explanation of the near constancy of the observed mass flux the question remains: how is the wind accelerated to high speed - i.e. $700-900 \mathrm{~km} \mathrm{~s}^{-1}$. Leer and Holzer (1980) show that a high speed wind can be obtained if the energy per particle is high. In the context of a two-fluid proton electron solar wind there are two possibilities of maximizing the energy per particle. 
First consider the position of heat addition. As Leer and Holzer point out energy added beyond the critical point will not increase the coronal density scale height but rather go almost entirely into accelerating the wind to high speed. This is also borne out in the Hansteen and Leer (1995) study in which consistent models of the chromosphere corona solar wind system are constructed and where the position of energy addition is varied in a systematic manner. In the Hansteen and Leer paper it is shown that the asymptotic flow velocity increases, the coronal proton temperature increases, and the coronal base density decreases as energy is added further and further out in the corona.

Secondly, we can change the asymptotic flow speed by changing the ratio of heat inserted into protons versus that inserted in electrons. Electrons conduct heat more efficiently than protons and energy inserted in the electron fluid will tend to flow back to the chromosphere rather than into accelerating the wind. An increase in the energy deposited into electrons will increase the the coronal electron temperature and the coronal (electron) density while decreasing the asymptotic flow speed. These models also show that due the high electron conductivity it is very difficult to achieve models where the electron temperature in the corona is much above say $1.5 \mathrm{MK}$. The protons may have much higher temperatures.

In these models, with low electron temperatures, the electric field plays a minor role for the proton force balance. If helium is introduced in these models the electron density in the corona decreases more rapidly, but neither Coulomb collisions nor the effects on the electric field play an important role. Hence, helium plays a less important role in regulating the solar wind proton flux than has has been predicted by (Leer and Holzer, 1991; Bürgi, 1992; Leer et al., 1992); in these models the presence of a significant coronal helium abundance could increase the electric field and thereby regulate the proton flux (see Hansteen et al. 1997 for a thorough discussion).

As an aside we note that the treatment of the proton heat flux (Olsen and Leer, 1996; Evje and Leer, 1997) of the coronal hole will also determine the asymptotic flow speed. When heat is added to the collisionless proton gas, in the outer corona, we can obtain high asymptotic flow speed. The dependence of the asymptotic flow speed on how and where energy is added to the flow is interesting to study, but that these effects are of less fundamental importance than the issues raised by the present paper.

\section{Summary}

In a corona, where the heat is deposited over a length scale that is larger than the density scale height, most of the energy is lost in the solar wind. In such models the solar wind mass flux is roughly proportional to the energy flux deposited in the corona. In order to drive high speed streams a significant fraction of the energy flux must be added to the proton gas over a scale length of $1 R_{\odot}$ or more. We therefore expect high proton (and ion) temperatures, low electron temperatures and low electron densities in coronal hole observations obtained by the SOHO satellite.

Acknowledgments. This work was supported by the Norwegian Research Council (NFR) under contracts D.115828/431 and D.115831/431 and by NASA under contract W-18786.

\section{References}

Bürgi, A.: 1992, J. Geophys. Res. 97(A3), 3137

Evje, H. and Leer, E.: 1997, $a \alpha$, in press

Hammer, R.: 1982a, Astrophys. J. 259, 767

Hammer, R.: 1982b, Astrophys. J. 259, 779

Hansteen, V. H. and Leer, E.: 1995, J. Geophys. Res. $100($ A11), 21577

Hansteen, V. H., Leer, E., and Holzer, T. E.: 1997, A strophys. J. 482, 498

Leer, E. and Holzer, T. E.: 1980, J. Geophys. Res. 85(A9), 4681

Leer, E. and Holzer, T. E.: 1991, Ann. Geophysicae 9, 196

Leer, E., Holzer, T. E., and Shoub, E. C.: 1992, J. Geophys. Res. $97(\mathbf{A 6}), 8183$

McComas, D. J., Phillips, J. L., Bame, S. J., Gosling, J. T., Goldstein, B. E., and Neugebauer, M.: 1995, Space Sci. Rev. 72, 93

Olsen, E. L. and Leer: 1996, J. Geophys. Res. 101, 15591

Parker, E. N.: 1958, A strophys. J. 128, 664

Withbroe, G. L.: 1988, A strophys. J. 325, 442 